\title{
A LOCAL HOPF LEMMA FOR SOLUTIONS OF THE ONE DIMENSIONAL HEAT EQUATION
}

\author{
NORIAKI SUZUKI
}

\begin{abstract}
The boundary behavior of solutions of the heat equation (temperature functions) is investigated. It is proved that a temperature function is identically equal to zero if it vanishes of finite order at some lateral boundary point where it attains a local minimum.
\end{abstract}

$\S 1$.

In [1], Baouendi and Rothschild proved the following uniqueness theorem for harmonic functions: Let $h$ be a harmonic function in a ball $B$ which is continuous on the closure $\bar{B}$. Take a boundary point $x_{0} \in \partial B$ where the function $\left.h\right|_{\partial B}$ attains a local minimum. If $h$ vanishes of infinite order at $x_{0}$, then $h$ is identically equal to zero. They call this result a local Hopf lemma in relation with the classical Hopf lemma. (The classical Hopf lemma says that, if $h$ has a "minimum" at $x_{0} \in \partial B$, and vanishes "of one order" at $x_{0}$, then $h \equiv 0$ (cf. [4]).

In this note we consider a solution of the one dimensional heat equation

$$
u_{x x}=u_{t}
$$

on a domain in the Euclidean space $\mathbf{R} \times \mathbf{R}$ and discuss a thermic analogue of the above result on harmonic functions.

Our theorem is the following

THEOREM 1. Let $T>0$. Let $u(x, t)$ be a continuous function on the rectangle $[0, \pi] \times[0, T]$ and satisfy the heat equation in $(0, \pi) \times(0, T)$. Assume that there exists $\varepsilon_{0}$ with $0<\varepsilon_{0}<T$ such that

$$
|u(\pi, t)| \leq u(0, t) \quad \text { for } t \in\left[T-\varepsilon_{0}, T\right] .
$$

If, for every positive integer $N$,

$$
\lim _{x \rightarrow 0+} \frac{u(x, T)}{x^{N}}=0
$$

Received May 25, 1995. 
then $u(x, t) \equiv 0$ on $[0, \pi] \times\left[T-\varepsilon_{0}, T\right]$.

The outline of our proof depends on the method used in [1], but the details are necessarily different. In particular, we have to pay attention to the fact that a solution of the heat equation is not always analytic with respect to the time variable. For this reason, to obtain our assertion we need some condition which controls the behavior of $u$ at $x=\pi$, though it seemes to be apparently surplus (cf. the last paragraph in $\S 3$ ). The proof of Theorem 1 is given in $\S 4$. In $\S 5$, using the same method, we also show the following theorem, which may be more suitable for the name of local Hopf lemma.

THEOREM 2. Let $T>2$ and $u(x, t)$ be same as in Theorem 1. Assume that there exists $\varepsilon_{0}$ with $0<\varepsilon_{0}<T$ such that

$$
u(0, t) \geq 0 \quad \text { and } \quad u(\pi, t) \geq 0 \quad \text { for } t \in\left[T-\varepsilon_{0}, T\right]
$$

or

$$
u(0, t) \geq 0 \quad \text { and } \quad u(\pi, t) \leq 0 \quad \text { for } t \in\left[T-\varepsilon_{0}, T\right]
$$

If, for every positive integer $N$,

$$
\lim _{x \rightarrow 0+} \frac{u(x, T)}{x^{N}}=0 \quad \text { and } \quad \lim _{x \rightarrow 0+} \frac{u(\pi-x, T)}{x^{N}}=0,
$$

then $u(x, t) \equiv 0$ on $[0, \pi] \times\left[T-\varepsilon_{0}, T\right]$.

In the sequel, we shall call a solution of the heat equation a temperature function.

$\S 2$.

Let $u$ be a temperature function as in Theorem 1. For the present, we only assume (1) for $u$. It is well-known (cf. [6, p.106]) that, for each $(x, t) \in(0, \pi) \times(0, T)$,

$$
\begin{aligned}
u(x, t)= & \int_{0}^{\pi}[\theta(x-y, t)-\theta(x+y, t)] u(y, 0) d y \\
& +\int_{0}^{t} \varphi(x, t-s) u(0, s) d s+\int_{0}^{t} \varphi(\pi-x, t-s) u(\pi, s) d s
\end{aligned}
$$


where $\theta$ and $\varphi$ are non-negative functions defined by

$$
\begin{aligned}
& \theta(y, s)=\frac{1}{2 \pi}+\frac{1}{\pi} \sum_{n=1}^{\infty} e^{-n^{2} s} \cos n y, \\
& \varphi(y, s)=\frac{2}{\pi} \sum_{n=1}^{\infty} n e^{-n^{2} s} \sin n y,
\end{aligned}
$$

for $-\infty<y<\infty$ and $s>0$. Note that this representation holds for $t=T$ (cf. Lemma 4 below).

Now put

$$
\begin{aligned}
& f(s)= \begin{cases}u(0, s) & \text { on }\left[T-\varepsilon_{0}, T\right] \\
0 & \text { on }\left[0, T-\varepsilon_{0}\right)\end{cases} \\
& g(s)= \begin{cases}u(\pi, s) & \text { on }\left[T-\varepsilon_{0}, T\right] \\
0 & \text { on }\left[0, T-\varepsilon_{0}\right)\end{cases}
\end{aligned}
$$

and we write

$$
u=w_{1}+w_{2}+w_{3}
$$

where

$$
\begin{aligned}
w_{1}(x, t) & =\int_{0}^{t} \varphi(x, t-s) f(s) d s \\
w_{2}(x, t) & =\int_{0}^{t} \varphi(\pi-x, t-s) g(s) d s \\
w_{3}(x, t) & =\int_{0}^{T-\varepsilon_{0}}[\varphi(x, t-s) u(0, s)+\varphi(\pi-x, t-s) u(\pi, s)] d s \\
& +\int_{0}^{\pi}[\theta(x-y, t)-\theta(x+y, t)] u(y, 0) d y .
\end{aligned}
$$

Note also that all $w_{i}$ are temperature and that the above equalities hold for $t=T$.

LEMMA 1. There is a sequence $\left\{a_{j}\right\}_{j=1}^{\infty}$ of real numbers such that for every integer $N>0$,

$$
w_{1}(x, T)=\sum_{j=1}^{N} a_{j} x^{2 j-1}+O\left(x^{2 N+1}\right) \quad \text { as } x \downarrow 0
$$


if and only if for every interger $N>0$,

$$
\sum_{n=1}^{\infty} n^{2 N} \int_{0}^{T} e^{-n^{2}(T-s)} f(s) d s<\infty
$$

Moreover if (7) holds for all integers, we have

$$
a_{j}=\frac{(-1)^{j-1}}{(2 j-1) !} \sum_{n=1}^{\infty} \frac{2}{\pi} n^{2 j} \int_{0}^{T} e^{-n^{2}(T-s)} f(s) d s \quad \text { for all } j \in \mathbf{N} .
$$

Proof. To show "if" part, we fix an integer $N>0$. Using the Taylor expansion

$$
\begin{aligned}
\sin n x= & n x+\cdots+(-1)^{N-1} \frac{(n x)^{2 N-1}}{(2 N-1) !} \\
& +(-1)^{N} \frac{(n x)^{2 N}}{(2 N-1) !} \int_{0}^{1}(1-t)^{2 N-1} \sin n x t d t
\end{aligned}
$$

we have

$$
\begin{aligned}
w_{1}(x, T) & =\int_{0}^{T} \frac{2}{\pi}\left[\sum_{n=1}^{\infty} n^{2 N+1} e^{-n^{2}(T-s)} \sin n x\right] f(s) d s \\
& =a_{1} x+\cdots+a_{N} x^{2 N-1}+A_{N}
\end{aligned}
$$

where

$$
A_{N}=\frac{(-1)^{N} x^{2 N}}{(2 N-1) !} \int_{0}^{T} \frac{2}{\pi} \sum_{n=1}^{\infty} n^{2 N+1} e^{-n^{2}(T-s)}\left[\int_{0}^{1}(1-t)^{2 N-1} \sin n x t d t\right] f(s) d s .
$$

Since $|\sin n x t| \leq n x t,(7)$ gives $\left|A_{N}\right|=O\left(x^{2 N+1}\right)$. This implies that (6) holds for $N$.

To show "only if" part, we use the induction on $N$. Let $\delta>0$. Since $\varphi(x, T-s) \geq 0$ and $f(s) \geq 0$, we have

$$
\begin{aligned}
\frac{w_{1}(x, T)}{x} & =\int_{0}^{T} \frac{\varphi(x, T-s)}{x} f(s) d s \\
& \geq \int_{0}^{T-\delta} \frac{\varphi(x, T-s)}{x} f(s) d s \\
& =\int_{0}^{T-\delta} \frac{2}{\pi} \sum_{n=1}^{\infty} n e^{-n^{2}(T-s)} \frac{\sin n x}{x} f(s) d s .
\end{aligned}
$$


Letting $x \downarrow 0$ and next $\delta \downarrow 0$, we have

$$
a_{1} \geq \int_{0}^{T} \frac{2}{\pi} \sum_{n=1}^{\infty} n^{2} e^{-n^{2}(T-s)} f(s) d s .
$$

This shows (7) for $N=1$. Moreover by the dominated convergence theorem, we have

$$
a_{1}=\frac{2}{\pi} \sum_{n=1}^{\infty} n^{2} \int_{0}^{T} e^{-n^{2}(T-s)} f(s) d s .
$$

Suppose that (7) holds for $N \geq 1$ and (8) holds for $j=1,2, \cdots, N$. We shall prove (7) for $N+1$. The argument is similar to the previous one. We first remark that for $x>0, n \geq 1$,

$$
\int_{0}^{1}(1-t)^{2 N-1} \sin n x t d t=\frac{1}{n x} \int_{0}^{1}(2 N-1)(1-t)^{2 N-2}(1-\cos n x t) d t \geq 0 .
$$

By (9) and the above remark, we see

$$
\begin{aligned}
& (-1)^{N} \frac{w_{1}(x, T)-\sum_{j=1}^{N} a_{j} x^{2 j-1}}{x^{2 N+1}} \\
& =\frac{1}{(2 N-1) !} \int_{0}^{T} \frac{2}{\pi} \sum_{n=1}^{\infty} n^{2 N+1} e^{-n^{2}(T-s)}\left[\int_{0}^{1}(1-t)^{2 N-1} \frac{\sin n x t}{x} d t\right] f(s) d s \\
& \geq \frac{1}{(2 N-1) !} \int_{0}^{T-\delta} \frac{2}{\pi} \sum_{n=1}^{\infty} n^{2 N+1} e^{-n^{2}(T-s)}\left[\int_{0}^{1}(1-t)^{2 N-1} \frac{\sin n x t}{x} d t\right] f(s) d s
\end{aligned}
$$

for any $\delta>0$. Letting $x \downarrow 0$, we obtain

$$
\begin{aligned}
& (-1)^{N} a_{N+1} \\
& \geq \frac{1}{(2 N-1) !} \int_{0}^{T-\delta} \frac{2}{\pi} \sum_{n=1}^{\infty} n^{2 N+2} e^{-n^{2}(T-s)}\left[\int_{0}^{1} t(1-t)^{2 N-1} d t\right] f(s) d s \\
& \quad=\frac{1}{(2 N+1) !} \int_{0}^{T-\delta} \frac{2}{\pi} \sum_{n=1}^{\infty} n^{2 N+2} e^{-n^{2}(T-s)} f(s) d s .
\end{aligned}
$$

Since $\delta>0$ is arbitrary, we see that (7) holds for $N+1$. The dominated convergence theorem again shows

$$
a_{N+1}=\frac{(-1)^{N}}{(2 N+1) !} \frac{2}{\pi} \sum_{n=1}^{\infty} n^{2 N+2} \int_{0}^{T} e^{-n^{2}(T-s)} f(s) d s .
$$

This completes the proof of Lemma 1. 
As for $w_{2}$ we have

LEMMA 2. There exists a sequence $\left\{b_{j}\right\}_{j=1}^{\infty}$ of real numbers such that, for every integer $N>0$,

$$
w_{2}(x, T)=\sum_{j=1}^{N} b_{j} x^{2 j-1}+O\left(x^{2 N+1}\right) \quad \text { as } x \downarrow 0 .
$$

Moreover if $w_{1}$ satisfies (6) for all $N>0$, then we see

$$
a_{j}+b_{j}=\frac{(-1)^{j-1}}{(2 j-1) !} \frac{2}{\pi} \sum_{n=1}^{\infty} n^{2 j} \int_{0}^{T} e^{-n^{2}(T-s)}\left(f(s)+(-1)^{n-1} g(s)\right) d s .
$$

Proof. To show (10) we use another expression of $\varphi$. Let $k(x, t)$ be the fundamental function of the heat equation, i.e.,

$$
k(x, t)=\frac{1}{\sqrt{4 \pi t}} e^{-x^{2} / 4 t} \quad(x \in \mathbf{R}, t>0) .
$$

Then

$$
\varphi(\pi-x, T-s)=\sum_{n=-\infty}^{\infty} \frac{\pi-x}{T-s} k(-x+(2 n+1) \pi, T-s)
$$

(see $[5, \mathrm{p} .86])$. Remark that the series converges uniformly for $(x, s) \in$ $[0, \pi / 2] \times(0, T)$. Hence putting

$$
b_{j}=\frac{1}{(2 j-1) !} \int_{0}^{T} \frac{\partial^{2 j-1} \varphi}{\partial x^{2 j-1}}(\pi, T-s) g(s) d s
$$

and observing

$$
\frac{\partial^{2 j} \varphi}{\partial x^{2 j}}(\pi, T-s) \equiv 0
$$

we have (10).

Moreover if (6) holds for all $N$, then by the proof of Lemma 1 and the assumption (1), we see

$$
\begin{aligned}
w_{2}(x, T) & =\int_{0}^{T} \frac{2}{\pi}\left[\sum_{n=1}^{\infty} n e^{-n^{2}(T-s)} \sin n(\pi-x)\right] g(s) d s \\
& =\int_{0}^{T} \frac{2}{\pi}\left[\sum_{n=1}^{\infty} n e^{-n^{2}(T-s)}(-1)^{n-1} \sin n x\right] g(s) d s \\
& =b_{1} x+\cdots+b_{N} x^{2 N-1}+O\left(x^{2 N+1}\right),
\end{aligned}
$$


where

$$
b_{j}=\frac{(-1)^{j-1}}{(2 j-1) !} \sum_{n=1}^{\infty} \frac{2}{\pi} n^{2 j} \int_{0}^{T}(-1)^{n-1} e^{-n^{2}(T-s)} g(s) d s \quad \text { for all } j \in \mathbf{N}
$$

This and (8) show (11) immediately.

The last function $w_{3}$ has the following expansion.

LEMMA 3. We have

$$
w_{3}(x, T)=\sum_{j=1}^{N} c_{j} x^{2 j-1}+O\left(x^{2 N+1}\right) \quad \text { as } x \downarrow 0
$$

for every integer $N>0$, where

$$
\left|c_{j}\right| \leq \frac{C^{j} j !}{(2 j-1) !} \quad \text { for all } j \in \mathbf{N}
$$

with some constant $C>0$.

Proof. Recall that

$$
\begin{aligned}
w_{3}(x, T)=\int_{0}^{T-\varepsilon_{0}}[\varphi(x, T-s) & u(0, s)+\varphi(\pi-x, T-s) u(\pi, s)] d s \\
& +\int_{0}^{\pi}[\theta(x-y, T)-\theta(x+y, T)] u(y, 0) d y
\end{aligned}
$$

Similarly to (9), we have

$$
\begin{aligned}
\int_{0}^{T-\varepsilon_{0}}[\varphi(x, T & -s) u(0, s)+\varphi(\pi-x, T-s) u(\pi, s)] d s \\
& =\int_{0}^{T-\varepsilon_{0}} \frac{2}{\pi} \sum_{n=1}^{\infty} n e^{-n^{2}(T-s)} \sin n x\left[u(0, s)-(-1)^{n} u(\pi, s)\right] d s \\
& =\sum_{j=1}^{N} \alpha_{j} x^{2 j-1}+O\left(x^{2 N+1}\right),
\end{aligned}
$$

where

$$
\alpha_{j}=\frac{(-1)^{j-1}}{(2 j-1) !} \frac{2}{\pi} \sum_{n=1}^{\infty} n^{2 j} \int_{0}^{T-\varepsilon_{0}} e^{-n^{2}(T-s)}\left[u(0, s)-(-1)^{n} u(\pi, s)\right] d s .
$$


Therefore, for any $j \in \mathbf{N}$,

$$
\begin{aligned}
\left|\alpha_{j}\right| & \leq \frac{2}{\pi} \int_{0}^{T}(|u(0, s)|+|u(\pi, s)|) d s \frac{1}{(2 j-1) !} \sum_{n=1}^{\infty} n^{2 j} e^{-n^{2} \varepsilon_{0}} \\
& \leq C_{1} \frac{1}{(2 j-1) !} \int_{0}^{\infty} x^{2 j} e^{-\varepsilon_{0} x^{2} / 4} d x \leq C_{1} \frac{j !}{(2 j-1) !}\left(\frac{4}{\varepsilon_{0}}\right)^{j}
\end{aligned}
$$

with some constant $C_{1}>0$.

Using Taylor expansion of $\sin n x$ again, we have

$$
\begin{aligned}
& \int_{0}^{\pi} {[\theta(x-y, T)-\theta(x+y, T)] u(y, 0) d y } \\
& \quad=\int_{0}^{\pi}\left[\frac{2}{\pi} \sum_{n=1}^{\infty} e^{-n^{2}} \sin n x \sin n y\right] u(y, 0) d y=\sum_{j=1}^{N} \beta_{j} x^{2 j-1}+O\left(x^{2 N+1}\right),
\end{aligned}
$$

where

$$
\beta_{j}=\frac{(-1)^{j-1}}{(2 j-1) !} \frac{2}{\pi} \int_{0}^{\pi}\left[\sum_{n=1}^{\infty} n^{2 j} e^{-n^{2}} \sin n x\right] u(y, 0) d y
$$

Thus

$$
\left|\beta_{j}\right| \leq \frac{2}{\pi} \int_{0}^{\pi}|u(y, 0)| d y \frac{1}{(2 j-1) !} \sum_{n=1}^{\infty} n^{2 j} e^{-n^{2}} \leq C_{2} \frac{j ! 4^{j}}{(2 j-1) !} .
$$

Since $c_{j}=\alpha_{j}+\beta_{j}$, we have the desired inequlity.

$\S \mathbf{3}$.

In this section we note two lemmas concerning an extension of temperature functions. Here the mean-value characterization for temperature functions plays an essential role (see [5, p.408]).

LEMMA 4. Let $v$ be a temperature function on a rectangle $R=(0, \pi) \times$ $(a, b)$ and continuous on $\bar{R}$. Then $v$ has an extension on $(0, \pi) \times(a, \infty)$ as a temperature function.

Proof. We put $v(0, t)=v(\pi, t)=0(t \geq b)$. Then

$$
\begin{aligned}
\tilde{v}(x, t)=\int_{0}^{\pi} \theta(x- & y, t)-\theta(x+y, t)] v(y, 0) d y \\
& +\int_{a}^{t} \varphi(x, t-y) v(0, y) d y+\int_{a}^{t} \varphi(\pi-x, t-y) v(\pi, y) d y
\end{aligned}
$$

is a desired function. 
LEMMA 5. Let $R$ and $v$ be as in Lemma 4 . If

$$
v(x, b)=v(0, s)=v(\pi, s)=0
$$

for $x \in(0, \pi)$ and $s \in(a, b)$. Then $v \equiv 0$ on $R$.

Proof. Since $v(0, s)=v(\pi, s)=0$, we see the following function $v_{1}$ defined by

$$
v_{1}(x, s)= \begin{cases}-v(2 \pi-x, s) & \text { for } \pi \leq x \leq 2 \pi \\ v(x, s) & \text { for } 0 \leq x \leq \pi \\ -v(-x, s) & \text { for }-\pi \leq x \leq 0\end{cases}
$$

is temperature on $(-\pi, 2 \pi) \times(a, b)$ (cf. $[6$, p.115]). Repeating this reflection, we have a temperature function $\tilde{v}$ on $\mathbf{R} \times(a, b)$, which is an extension of $v$. Since $\tilde{v}$ is bounded and $\tilde{v}(x, b)=0$ for all $x \in \mathbf{R}$, we have $\tilde{v} \equiv 0$ (cf. [6, p.183] or [2, p.149]). Thus $v \equiv 0$ on $R$.

Here we remark that there is a temperature function $v \neq \equiv$ on $R$ such that it is continuous on $\bar{R}$ and satisfying

$$
v(x, b)=v(x, a)=v(0, s)=0
$$

for all $x \in(0, \pi)$ and all $s \in(a, b)$ (see [3, Corollary 3]).

$\S 4$.

Now we give a proof of Theorem 1. By (2), (5), (10) and (12), we see that (6) holds and hence by (13)

$$
\left|a_{j}+b_{j}\right|=\left|c_{j}\right| \leq \frac{C^{j} j !}{(2 j-1) !}
$$

with some constant $C>0$. Set

$$
\begin{aligned}
& d_{j}=\frac{1}{(2 j-1) !} \frac{2}{\pi} \sum_{n=1}^{\infty}(2 n)^{2 j} \int_{0}^{T} e^{-(2 n)^{2}(T-s)}(f(s)-g(s)) d s \\
& e_{j}=\frac{1}{(2 j-1) !} \frac{2}{\pi} \sum_{n=1}^{\infty}(2 n-1)^{2 j} \int_{0}^{T} e^{-(2 n-1)^{2}(T-s)}(f(s)+g(s)) d s
\end{aligned}
$$


Then all terms in $d_{j}$ and $e_{j}$ are positive and $d_{j}+e_{j}=\left|b_{j}+a_{j}\right|$ by (11). For every $\eta>0$,

$$
\begin{aligned}
\frac{C^{j} j !}{(2 j-1) !} & \geq\left|c_{j}\right| \\
& \geq \frac{1}{(2 j-1) !} \frac{2}{\pi} \sum_{n=1}^{\infty}(2 n)^{2 j} \int_{T-\eta}^{T} e^{-(2 n)^{2}(T-s)}(f(s)-g(s)) d s \\
& \geq \frac{1}{(2 j-1) !} \frac{2}{\pi} \sum_{n=1}^{\infty}(2 n)^{2 j} e^{-(2 n)^{2} \eta} \int_{T-\eta}^{T}(f(s)-g(s)) d s \\
& \geq C_{2} \frac{j !}{(2 j-1) !}\left(\frac{1}{4 \eta}\right)^{j !} \int_{T-\eta}^{T}(f(s)-g(s)) d s,
\end{aligned}
$$

which gives

$$
\int_{T-\eta}^{T}(f(s)-g(s)) d s \leq C_{2}^{-1}(4 C \eta)^{j} .
$$

Take $\eta_{1}$ such that $4 C \eta_{1}<1$. Then letting $j \rightarrow \infty$, we have

$$
\int_{T-\eta_{1}}^{T}(f(s)-g(s)) d s=0
$$

and hence $f(s)-g(s)=0$ for $s \in\left[T-\eta_{1}, T\right]$. Similarly, from the estimate of $e_{j}$ it follows that there is $\eta_{2}>0$ such that $f(s)+g(s)=0$ for $s \in\left[T-\eta_{2}, T\right]$. Therefore

$$
u(0, s)=u(\pi, s)=0 \quad \text { for all } s \in\left[T-\eta_{0}, T\right]
$$

where $\eta_{0}=\min \left\{\eta_{1}, \eta_{2}\right\}$. By the reflextion

$$
\tilde{u}(x, t)= \begin{cases}u(x, t) & (x, t) \in[0, \pi] \times\left(T-\eta_{0}, T\right] \\ -u(-x, t) & (x, t) \in[-\pi, 0] \times\left(T-\eta_{0}, T\right]\end{cases}
$$

and Lemma 4 , we may assume that $u$ is a temperature function on a neighborhood of $(0, T)$. Since $u(x, T)$ is an analytic function of $x$ (cf. [2, p.118]), condition (2) implies $u(x, T)=0$ for $x \in[0, \pi]$. Thus Lemma 5 shows that $u \equiv 0$ on $[0, \pi] \times\left[T-\eta_{0}, T\right]$.

Now let

$$
\varepsilon_{1}=\sup \left\{\varepsilon>0 ; \varepsilon<\varepsilon_{0}, u \equiv 0 \text { on }[0, \pi] \times[T-\varepsilon, T]\right\} .
$$

Assume that $\varepsilon_{1}<\varepsilon_{0}$. Then remarking $u\left(x, T-\varepsilon_{1}\right)=0(x \in[0, \pi])$ and making the same argument for $u$ on $[0, \pi] \times\left[0, T-\varepsilon_{1}\right]$, we have $u \equiv 0$ on $[0, \pi] \times\left[T-\varepsilon_{1}-\eta, T-\varepsilon_{1}\right]$ for some $\eta>0$, which contradicts the fact that $\varepsilon_{1}$ is the supremum. Hence $\varepsilon_{1}=\varepsilon_{0}$ and we have in the end $u \equiv 0$ on $[0, \pi] \times\left[T-\varepsilon_{0}, T\right]$. 
$\S 5$.

To show Theorem 2, let $f$ and $g$ be same as defined in $\S 2$ and let $u(x, T)=w_{1}(x, T)+w_{2}(x, T)+w_{3}(x, T)$ be as in (5). Put $\tilde{w}_{1}(x, T)=$ $w_{2}(\pi-x, T), \tilde{w}_{2}(x, T)=w_{1}(\pi-x, T)$ and $\tilde{w}_{3}(x, T)=w_{3}(\pi-x, T)$, then

$$
u(\pi-x, T)=\tilde{w}_{1}(x, T)+\tilde{w}_{2}(x, T)+\tilde{w}_{3}(x, T) .
$$

By the arguments in $\S 2$ and our assumption (4), we have

$$
\begin{array}{ll}
w_{1}(x, T)=\sum_{j=1}^{N} a_{j} x^{2 j-1}+O\left(x^{2 N+1}\right) & \text { as } x \downarrow 0 \\
w_{2}(x, T)=\sum_{j=1}^{N} b_{j} x^{2 j-1}+O\left(x^{2 N+1}\right) & \text { as } x \downarrow 0 \\
w_{3}(x, T)=\sum_{j=1}^{N} c_{j} x^{2 j-1}+O\left(x^{2 N+1}\right) & \text { as } x \downarrow 0 \\
\tilde{w}_{1}(x, T)=\sum_{j=1}^{N} \tilde{a}_{j} x^{2 j-1}+O\left(x^{2 N+1}\right) & \text { as } x \downarrow 0 \\
\tilde{w}_{2}(x, T)=\sum_{j=1}^{N} \tilde{b}_{j} x^{2 j-1}+O\left(x^{2 N+1}\right) & \text { as } x \downarrow 0 \\
\tilde{w}_{3}(x, T)=\sum_{j=1}^{N} \tilde{c}_{j} x^{2 j-1}+O\left(x^{2 N+1}\right) & \text { as } x \downarrow 0
\end{array}
$$

and

$$
\begin{gathered}
a_{j}+b_{j}=\frac{1}{(2 j-1) !} \frac{2}{\pi} \sum_{n=1}^{\infty} n^{2 j} \int_{0}^{T} e^{-n^{2}(T-s)}\left(f(s)+(-1)^{n-1} g(s)\right) d s \\
\tilde{a}_{j}+\tilde{b}_{j}=\frac{1}{(2 j-1) !} \frac{2}{\pi} \sum_{n=1}^{\infty} n^{2 j} \int_{0}^{T} e^{-n^{2}(T-s)}\left(g(s)+(-1)^{n-1} f(s)\right) d s \\
\left|c_{j}\right| \text { and }\left|\tilde{c}_{j}\right| \leq \frac{C^{j} j !}{(2 j-1) !} \quad \text { for all } j \in \mathbf{N}
\end{gathered}
$$

with some constant $C>0$. Assume that (3) (resp. $\left.(3)^{\prime}\right)$ holds. Then estimating

$$
\left|a_{j}+b_{j}+\tilde{a}_{j}+\tilde{b}_{j}\right|=\left|c_{j}+\tilde{c}_{j}\right| \quad\left(\text { resp. }\left|a_{j}+b_{j}-\left(\tilde{a}_{j}+\tilde{b}_{j}\right)\right|=\left|c_{j}-\tilde{c}_{j}\right|\right)
$$

as in (14), we have $f(s)+g(s)=0$ (resp. $f(s)-g(s)=0$ ) for $s \in[T-\eta, T]$ with some $\eta>0$. Similar reason as in $\S 4$, we obtain Theorem 2 . 


\section{REFERENCES}

[1] M. S. Baouendi and L. P. Rothschild, A local Hopf lemma and unique continuation for harmonic functions, International Math. (in Duke Math. J.), Reserch Notices, 8 (1993), 245-251.

[2] J. R. Cannon, The one-dimensional heat equation, Addison-Wesley, 1984.

[3] B. F. Jones, Jr., A fundamental solution for the heat equation which is supported in a strip, J. of Math. Anal. and Appli., 60 (1977), 314-324.

[4] M. H. Protter and H. F. Weinberger, Maximum principles in differential equations, Springer, 1984.

[5] N. A. Watson, A theory of subtemperatures in several variables, Proc. London Math. Soc., 26 (1973), 385-417.

[6] D. V. Widder, The heat equation, Academic Press, 1975.

Graduate School of Polymathematics

Nagoya University

Chikusa-ku, Nagoya 464-01

Japan

nsuzuki@math.nagoya-u.ac.jp 